\title{
A mildly painful wrist mass
}

\author{
Wai Kan Tsang • Kwok Fai Godfrey Tam
}

Published online: 7 September 2013

(C) ISS 2013

\begin{abstract}
Answer
Familial tumoral calcinosis
\end{abstract}

\section{Discussion}

Familial tumoral calcinosis (FTC) is a rare autosomal recessive disease characterized by progressive deposition of periarticular calcifications $[1,2]$. It can be divided into hyperphosphatemic and normophosphatemic types depending on the serum phosphate level [3]. In the hyperphosphatemic type, the GALNT3 gene, which codes for a glycosyltransferase and FGF23 gene that codes for a phosphaturic protein, have been identified as the cause of phosphate dysregulation $[3,4]$. In the less common normophosphatemic type, SAMD9 gene mutation causes dysregulation of endothelial cell and

The case presentation can be found at doi:10.1007/s00256-013-1710-8.

W. K. Tsang $(\bowtie)$

Department of Radiology \& Nuclear Medicine, Tuen Mun Hospital,

Tuen Mun, New Territories, Hong Kong

e-mail: tsang_k@yahoo.com.hk

\section{K. F. G. Tam}

Department of Radiology, North District Hospital, Sheung Shui,

New Territories, Hong Kong fibroblast function. A positive family history is present in $30-40 \%$ of patients while the rest are sporadic [4]. FTC is most common in patients of African decent [1]. Typically, patients present in the first two decades of life with solitary or multiple painless periarticular "tumors" that reduce range of motion in the involved joints [2]. The periarticular swelling is painless unless there is nerve impingement. The overlying skin is usually intact. Occasionally, soft tissue ulceration with extrusion of chalky white material can be seen.

Radiographically, lobulated, variably calcified, juxtaarticular masses in bursal distribution can be seen (Figs. 1, 2 and 3a). Extensor surfaces are commonly involved, with descending frequency of distribution at hip, elbow, shoulder, foot, and wrist. Less commonly, they happen in extraarticular regions without naturally occurring bursa including scalp, larynx, spine, and sacrum, where there is synovial metaplasia forming bursa-like structures in response to trauma [2, 5]. Sedimentation sign, which represents fluid-fluid levels caused by calcium layering, is classical [6] (Fig. 3b,c). Although the sedimentation sign is usually best appreciated in cross-sectional imaging, sometimes it can be evident only in ultrasound [7]. On CT imaging, lobulated cystic calcification that communicates with the bursa can be demonstrated. The appearance of homogeneity suggests a reduced metabolic activity and lower likelihood of growth $[8,9]$. There should not be any erosion or destruction of adjacent bones. On MRI, FTC has a variegated appearance [2, 10]. In T1W 
Fig. 1 Right wrist radiographs taken on presentation. $\mathbf{a} \mathrm{AP}$ and $\mathbf{b}$ lateral view show lobulated calcified periarticular lesions around the right distal ulna (white arrowheads). There is no associated bone destruction or erosion
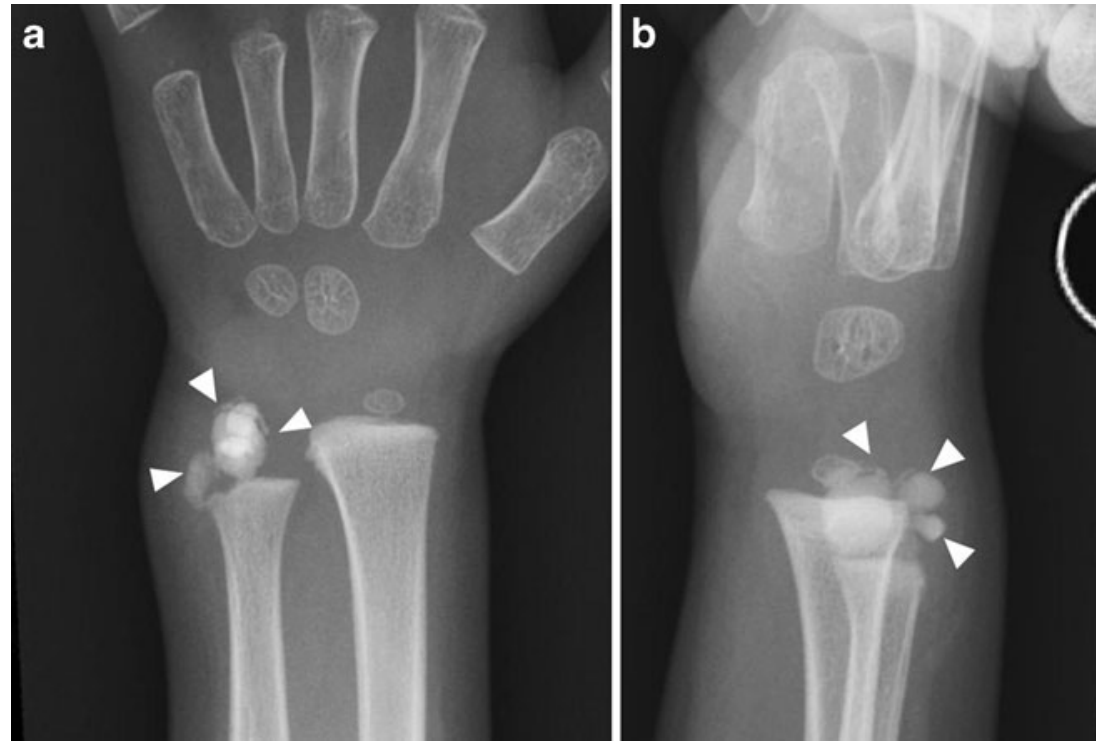

images, the calcification is low in signal. In T2W sequence, the calcification can either be diffusely hypointense, or with

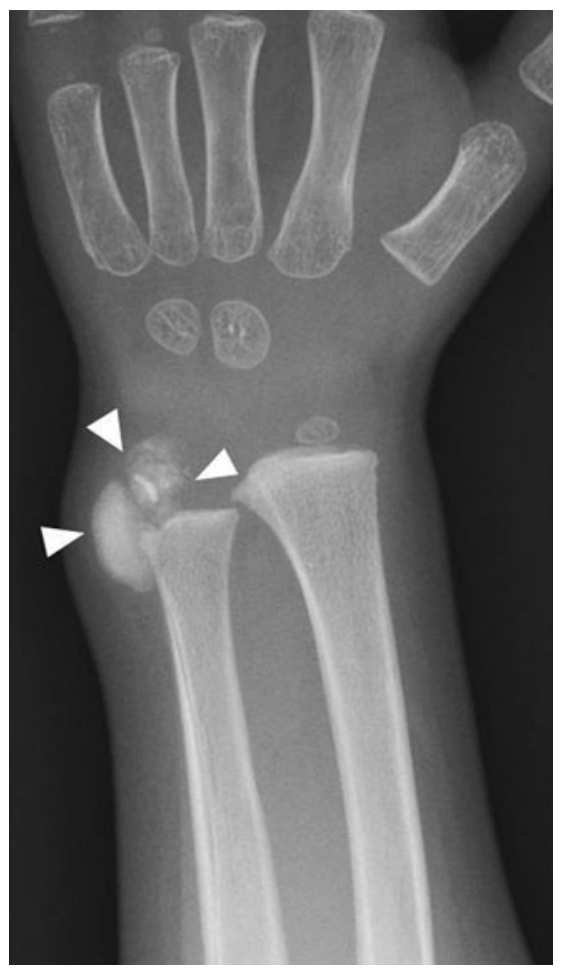

Fig. 2 Right wrist AP radiograph taken 10 days following initial presentation shows a rapid change in the appearance of the lesions hyperintense nodular pattern with alternating areas of high signal and signal void [2].

The diagnosis of FTC is one of exclusion. Apart from the periarticular masses, the clinical history of the patient is unremarkable. Family history can be present. Regarding the laboratory result, phosphate and 1,25-dihydroxy-vitamin D levels elevate occasionally, while calcium, parathyroid hormone, glomerular filtration, and antibodies level, should be normal [2].

Apart from FTC, other causes of periarticular calcification can be divided into metastatic calcification and dystrophic calcification. Concerning the periarticular calcification, there is no radiological or histological difference between them and FTC. The way to differentiate these mimics from FTC is by clinical history, serum chemistry levels, and imaging findings at other body parts [2]. Metastatic calcification usually results in generalized calcium deposition in normal tissues. It is associated with abnormal calcium and/or phosphate levels [2]. The most common cause is chronic renal failure, in which history and serum chemistry are essential for diagnosis. Features of renal osteodystrophy in the rest of the body (e.g. rugger-jersey spine) are useful. In contrast, dystrophic calcification is caused by calcium deposition in abnormal tissues. The most common cause is connective tissue diseases, followed by neoplastic and degenerative diseases. The serum chemistry levels in those patients are normal [2]. 

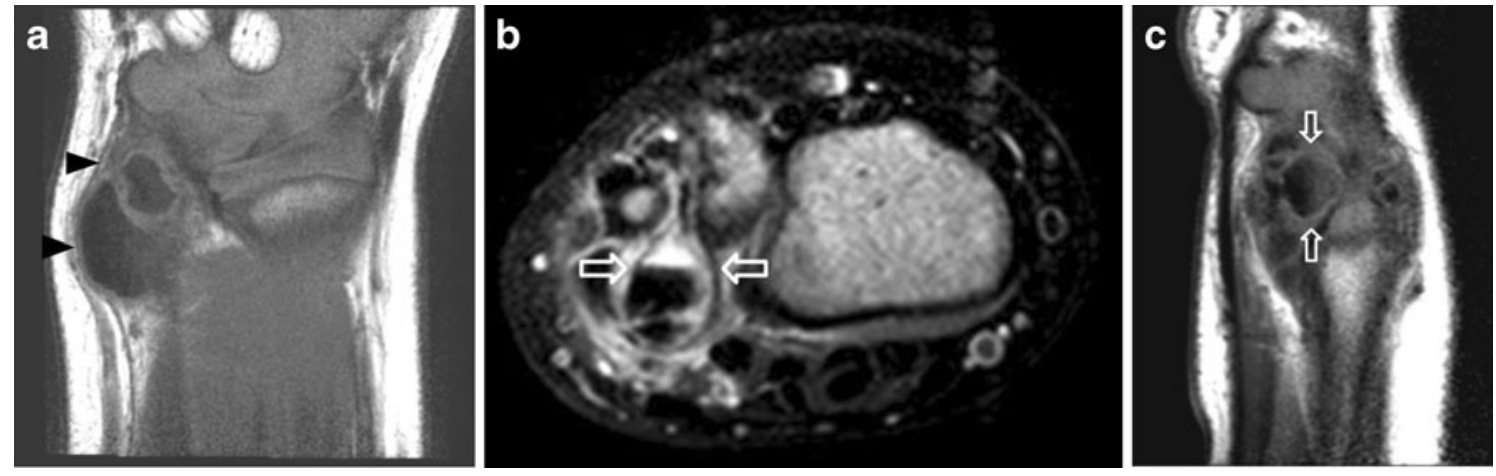

Fig. 3 MR images taken 2 weeks after presentation. a Coronal T1-weighted image shows lobulated lesions around the distal ulna with homogenous low signal (arrowheads), b axial T2-weighted fat-suppression image, and c sagittal T1-weighted enhanced image show sedimentation sign (empty arrows)

Conflict of interest The authors declare that they have no conflicts of interest.

\section{References}

1. Jose J, Fitcher B, Clifford PD. Familial tumoral calcinosis. Am J Orthop. 2010;39(10):E111-3.

2. Olsen KM, Chew FS. Tumoral calcinosis: pearls, polemics, and alternative possibilities. RadioGraphics. 2006;26:871-85.

3. Topaz $\mathrm{O}$, Indelman $\mathrm{M}$, Chefetz I, et al. A deleterious mutation in SAMD9 causes normophosphatemic familial tumoral calcinosis. Am J Hum Genet. 2006;79(4):759-64.

4. Joseph L, Hing SN, Presneau N, et al. Familial tumoral calcinosis and hyperostosis-hyperphosphataemia syndrome are different manifestations of the same disease: novel missense mutations in GALNT3. Skeletal Radiol. 2010;39(1):63-8.

5. Slavin RE, Wen J, Barmada A. Tumoral calcinosis-a pathogenetic overview: a histological and ultrastructural study with a report of two new cases, one in infancy. Int J Surg Pathol. 2012;20(5):462-73.

6. Hug I, Guncaga J. Tumoral calcinosis with sedimentation sign. Br J Radial. 1974;47:734-6.

7. Chakarun CJ, Talkin B, White EA, Romero M, Ralls PW. Tumoral calcinosis: sonographic sedimentation sign. J Clin Ultrasound. 2011;39(6):367-70.

8. Carmichael KD, Bynum JA, Evans E. Familial tumoral calcinosis: a forty-year follow-up on one family. J Bone Joint Surg Am. 2009;91(3):664-7.

9. Yamaguchi T, Sugimoto T, Imai Y, et al. Successful treatment of hyperphosphatemic tumoral calcinosis with long-term acetazolamide. Bone. 1995;16(4 suppl):247S-50S.

10. Martinez S, Vogler JB, Harrelson JM, Lyles KW. Imaging of tumoral calcinosis: new observations. Radiology. 1990;174:215-22. 